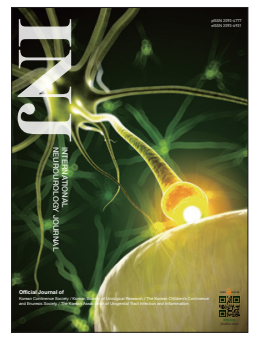

\title{
Effect of Alpha 1-Adrnoceptor Antagonists on Postsynaptic Sensitivity in Substantia Gelatinosa Neurons From Lumbosacral Spinal Cord in Rats Using Slice Patch-Clamp Technique for mEPSC
}

\author{
Daisuke Uta ${ }^{1,2 *}$, Tsuyoshi Hattori ${ }^{3 *}$, Megumu Yoshimura ${ }^{2,4,5}$ \\ ${ }^{1}$ Department of Applied Pharmacology, Faculty of Pharmaceutical Sciences, Toyama, Japan \\ ${ }^{2}$ Department of Integrative Physiology, Graduate School of Medical Sciences, Kyushu University, Fukuoka, Japan \\ ${ }^{3}$ Department of Medical Affairs, Asahi Kasei Pharma Co., Tokyo, Japan \\ ${ }^{4}$ Graduate School of Health Sciences, Kumamoto Health Science University, Kumamoto, Japan \\ ${ }^{5}$ Nogata Nakamura Hospital, Fukuoka, Japan
}

\begin{abstract}
Purpose: Alpha1-adrenoceptors participate in improving storage symptoms of male lower urinary tract symptoms (LUTS). However, the mechanism of action of these compounds remains unclear. To clarify the mechanism of the $\alpha 1$-adrenoceptor antagonists, the amplitude of miniature excitatory postsynaptic currents (mEPSCs) was analyzed in the lumbosacral spinal cord in rats.

Methods: Male adult Sprague-Dawley rats were used. Blind whole-cell patch-clamp recordings were performed on substantia gelatinosa (SG) neurons in spinal cord slice preparations. The amplitude of mEPSCs was recorded in individual SG neurons to which $\alpha 1$-adrenoceptors ( $100 \mu \mathrm{M}$ naftopidil, $100 \mu \mathrm{M}$ tamsulosin, and $30 \mu \mathrm{M}$ silodosin) were applied sequentially with intervening washout periods. Individual amplitudes were analyzed.

Results: Pearson correlation coefficients ( $r$ ) for the amplitudes of mEPSCs between the baseline and postadministration of $\alpha 1$ adrenoceptor antagonists indicated changes of the amplitude ranked in the order of naftopidil $(r=0.393)$, tamsulosin $(r=0.738)$, and silodosin $(r=0.944)$. Together, the $\alpha 1$-adrenoceptor antagonists yielded significant increases in the amplitude of mEPSCs in SG neurons ( $\mathrm{n}=108, \mathrm{P}=0.012$ ). However, the effects of each $\alpha 1$-adrenoceptor antagonist on the amplitude were as follows (relative to the baseline; $\mathrm{n}=36$ each): naftopidil, $\mathrm{P}=0.129$; tamsulosin, $\mathrm{P}=0.201$; and silodosin, $\mathrm{P}=0.005$. The rate of response to naftopidil for the outward current was relatively high among the $\alpha 1$-adrenoceptor blockers. An inward current was observed only with the naftopidil application.

Conclusions: Alpha1-adrenoceptor antagonists changed the amplitudes of mEPSCs in a subset of SG neurons in slices prepared from the L6-S1 levels of rat spine. Although the $\alpha 1$-adrenoceptor antagonists generated inward or outward currents in the SG neurons, different rates of response were observed with each antagonist. These results are important for understanding the mechanisms of action (at the spinal level) of $\alpha 1$-adrenoceptor antagonists for the storage symptoms of male LUTS.
\end{abstract}

Keywords: Adrenergic alpha-1 receptor antagonists; Excitatory postsynaptic potentials; Naftopidil; Substantia gelatinosa

Corresponding author: Daisuke Uta iD https://orcid.org/0000-0001-5644-1348 Department of Applied Pharmacology, Faculty of Pharmaceutical Sciences, University of Toyama, Toyama 930-0194, Japan

E-mail: daicarp@pha.u-toyama.ac.jp

Corresponding author: Tsuyoshi Hattori (iD https://orcid.org/0000-0001-6425-3945 Department of Medical Affairs, Asahi Kasei Pharma Co., Tokyo 100-0006, Japan E-mail: hattori.tg@om.asahi-kasei.co.jp

${ }^{*}$ Daisuke Uta and Tsuyoshi Hattori contributed equally to this study as co-first authors.

Submitted: December 18, 2019 / Accepted after revision: February 18, 2020
cC) (7) This is an Open Access article distributed under the terms of the Cre(c) ative Commons Attribution Non-Commercial License (https://creativecommons.org/licenses/by-nc/4.0/) which permits unrestricted non-commercial use, distribution, and reproduction in any medium, provided the original work is properly cited. 
- Grant/Fund Support: This work was supported by grants from the programs for Grants-in-Aid for Scientific Research (DU and MY) of the Ministry of Education, Science, Sports and Culture of Japan (Grant Numbers JP19K09323, JP15K08667, JP25860431, and JP21600005), and was partially supported by the MEXT-Supported Program for the Strategic Research Foundation at Private Universities, "Creation of 3D nano-micro structures and its application to biomimetics and medicine”, during 2015-2019 (DU, Grant Number S1511031). This study also was supported in part by the Asahi Kasei Pharma Corporation.

- Research Ethics: All experiments were performed in accordance with the "Guiding Principles for Care and Use of Animals in the Field of Physiological Sciences" of the Physiological Society of Japan and were approved by the local Animal Experiment Committee of the Kumamoto Health Science University and Kyushu University. All efforts were made to minimize animal suffering and the number of animals used for the studies.

- Conflict of Interest: TH is an employee of Asahi Kasei Pharma Corporation. The other authors have no potential conflicts of interest relevant to this article.

\section{- HIGHLIGHTS}

- Alpha1-adrenoceptor antagonists changed the amplitude of miniature excitatory postsynaptic currents in a subset of substantia gelatinosa neurons by slice patch-clamp technique in rat spinal cord.

- The magnitude of the changes ranked (in descending order) from naftopidil to tamsulosin to silodosin.

\section{INTRODUCTION}

Lower urinary tract symptoms (LUTS) consist of voiding, storage, and postmicturition symptoms [1]. Male LUTS is related to a variety of causes, including, for example, benign prostatic obstruction/benign prostatic hyperplasia, bladder dysfunction including overactive bladder, and nocturnal polyuria [2]. The prevalence of LUTS increases with age, and a considerable proportion of men are affected by LUTS [3]. To manage male LUTS pharmacologically, $\alpha 1$-adrenoceptor antagonists, $5 \alpha$-reductase inhibitors, phosphodiesterase 5 inhibitors, and plant extracts are prescribed. Among these treatments, $\alpha 1$-adrenoceptor blockers are the most established drugs and are widely prescribed and taken. $\alpha 1$-adrenoceptor antagonists act by relaxing smooth muscle in the urethra and prostate, resulting in a reduction of enhanced tonus or contractility, thus counteracting decreased urine flow rates [4]. Additionally, $\alpha 1$-adrenoceptors contribute to the improvement of storage symptoms [5], although the mechanism of action of these effects remains unclear. In rats subjected to conscious cystometry, tamsulosin, naftopidil, and silodosin were shown to prolong the micturition interval [6]. When isovolumetric cystometry was conducted in anesthetized animals, intrathecally administered naftopidil yielded increased inter-contraction intervals [7]. Interestingly, the effect of naftopidil was counteracted by bicuculline and/or glycine injected intrathecally [8]. Clinical studies have yielded contradictory results in comparisons between naftopidil and tamsulosin with evaluation by the International Prostate Symptom Score [9] and by urodynamic parameters [10]. The differences between the 2 studies were thought to reflect some aspect of the mode of ac- tion (e.g., a selectivity for subtypes of $\alpha 1$-adrenoceptors), but the actual basis of these differences remains unknown.

By using voltage-clamp recordings, it is possible to identify primary afferent information in substantia gelatinosa (SG, lamina II of Rexed) neurons [11], effects that are mediated by different primary afferent fibers $[12,13]$. The efficacy of synaptic transmission is determined by presynaptic neurotransmitter release probability and postsynaptic responsiveness, parameters that are evaluated by the frequency and amplitude of miniature excitatory or inhibitory postsynaptic currents (mEPSC or mIP$\mathrm{SC}$ ), respectively. A slice patch-clamp recording can measure miniature excitatory and inhibitory postsynaptic currents, which are thought to reflect the following details. The frequency of the current indicates presynaptic events, which correspond to changes in the probability of neurotransmitter release and changes in the number of synapses [14]. Meanwhile, the amplitude of the current indicates postsynaptic information, corresponding to changes in the number of receptors for neurotransmitters on the postsynaptic site. When outward/inward currents are observed, the recording neuron shows depolarization or hyperpolarization by activation of receptors for neurotransmitters at postsynaptic sites, yielding changes in membrane potential [15]. Analyses of frequency and amplitude distributions of mEPSCs permit determination of the loci of experimental manipulation (i.e., presynaptic and/or postsynaptic) [16]. The effects of naftopidil, tamsulosin, and silodosin on the frequency of excitatory synaptic currents at synaptic terminal sites in the spinal cord have been determined [17], and an explanation for the diversity of efficacy in treatment of storage symptoms by $\alpha 1$-adrenoceptor antagonists is being developed. 
Naftopidil inhibits the amplitudes of EPSCs evoked by dorsal root stimulation and increases the frequencies of mEPSCs [18]. However, the effect of naftopidil on the amplitudes of mEPSCs, meaning upregulation of sensitivity for depolarization of postsynaptic cells, has not yet been described (to our knowledge). Therefore, the goal of the present study was to clarify the effect of naftopidil on the amplitudes of the mEPSCs in SG neurons derived from lumbosacral spinal cord in rats and to explain the mechanism of action of $\alpha 1$-adrenoceptor antagonists in the treatment of storage symptoms.

\section{MATERIALS AND METHODS}

\section{Spinal Cord Slice Preparation}

The methods for obtaining slices of the adult rat spinal cord and for blind patch-clamp recordings from SG neurons have been described in detail elsewhere $[17,19]$. Briefly, adult male SpragueDawley rats (6-8 weeks old) were deeply anesthetized with urethane (1.2 g/ $\mathrm{kg}$, intraperitoneally), and a lumbosacral laminectomy then was performed. The lumbosacral segments of the spinal cord (L2-S3), with the associated ventral and dorsal roots, were removed and placed in ice-cold Krebs solution equilibrated with 95\% $\mathrm{O}_{2}-5 \% \mathrm{CO}_{2}$. The Krebs solution contained (in $\mathrm{mM}$ ): $\mathrm{NaCl}$ 117, $\mathrm{KCl}$ 3.6, $\mathrm{CaCl}_{2}$ 2.5, $\mathrm{MgCl}_{2}$ 1.2, $\mathrm{NaH}_{2} \mathrm{PO}_{4} 1.2, \mathrm{NaHCO}_{3} 25$, and glucose $11(\mathrm{pH}, 7.4)$. Immediately after removal of the spinal cord, the rats were killed by exsanguination under urethane anesthesia. The pia-arachnoid membrane was removed after cutting all of the ventral and dorsal roots. The spinal cord was mounted on a vibratome, and a 500- $\mu$ m-thick transverse slice with the attached dorsal root was cut. The slice was placed on a nylon mesh in the recording chamber in a volume of $0.5-\mathrm{mL}$ Krebs solution, and the slice was completely submerged in and perfused with Krebs solution saturated with $95 \% \mathrm{O}_{2}-5 \% \mathrm{CO}_{2}$ at $37^{\circ} \mathrm{C} \pm 1^{\circ} \mathrm{C}$ and a flow rate of $10-15 \mathrm{~mL} / \mathrm{min}$.

\section{Whole-Cell Patch-Clamp Recordings From SG Neurons}

The SG was easily discernible with transmitted illumination as a relatively translucent band across the dorsal horn in the transverse slice preparations (Fig. 1). Blind whole-cell voltage-clamp recordings were made from SG neurons, as previously described $[17,19]$. The patch pipettes were filled with a solution containing potassium gluconate solution (in $\mathrm{mM}$ ): K-gluconate 135, $\mathrm{KCl} 5, \mathrm{CaCl}_{2}$ 0.5, $\mathrm{MgCl}_{2}$ 2, EGTA 5, HEPES 5, and ATP$\mathrm{Mg} 5$ (pH 7.2). The tip resistance of the patch pipettes was 6-12 $\mathrm{M} \Omega$. Series resistance was assessed according to the response to

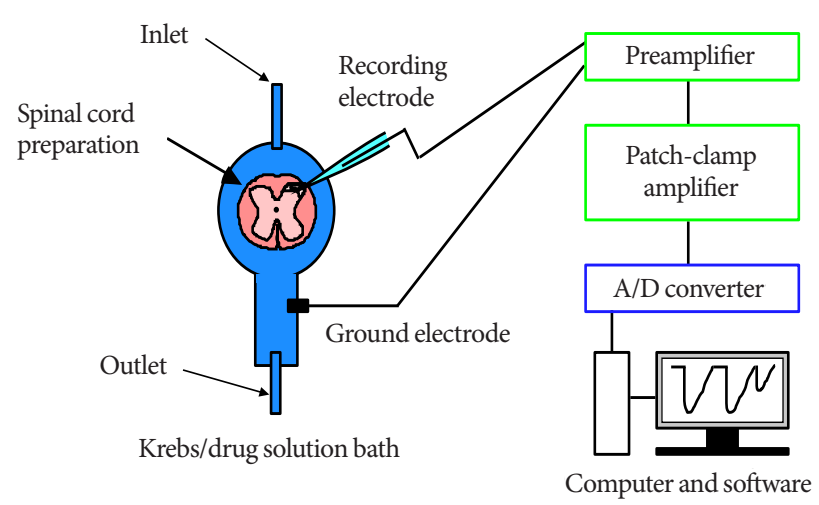

Fig. 1. The experimental method and design are illustrated. Blind whole-cell patch-clamp recording was performed in individual substantia gelatinosa neurons of an adult rat spinal dorsal horn. A/D, analog to digital.

a 5-mV hyperpolarizing step. This value was monitored during the recording session, and data were rejected if values changed by $>15 \%$. Signals were acquired with a patch-clamp amplifier (Axopatch 700A, Molecular Devices, Union City, CA, USA). The data were digitized with an $\mathrm{AD} / \mathrm{DA}$ converter (Digidata 1321A, Molecular Devices), stored on a personal computer using a data acquisition program (Clampex, version 9.0, Molecular Devices), and analyzed using a software package (Clampfit, version 9.0, Molecular Devices) (Fig. 1). Cell recordings were obtained in voltage-clamp mode at holding potentials of -70 $\mathrm{mV}$ to record EPSCs $[17,19]$.

\section{Drug Application}

1-[4-(2-methoxyphenyl) piperazinyl]-3-(1-naphthyloxy) propan-2-ol (naftopidil) (PubChem CID: 4418) (Asahi Kasei Pharma Co., Tokyo, Japan) was dissolved in $1 \%$ dimethyl sulfoxide (DMSO) (PubChem CID: 679) (Wako, Osaka, Japan) in Krebs solution. Tamsulosin and silodosin were dissolved in Krebs solution. All drugs were applied by sequential perfusion in a single cell with washout periods via a three-way stopcock without changes in the perfusion rate or temperature. The application schedule was as described previously [17].

\section{Statistical Analysis}

Statistical analysis was performed using JMP ver. 14 (SAS Institute, Cary, NC, USA), and P-values $<0.05$ were considered statistically significant. The analyses consisted of 4 sets of tests as described below. 


\section{Correlation of the amplitudes before and after administration of each $\alpha 1$-adrenoceptor antagonist in the SG neurons}

For individual SG neurons, the amplitude after administration of naftopidil, tamsulosin, or silodosin was plotted against amplitude before administration of the respective reagent. For each $\alpha 1$-adrenoceptor antagonist, correlations were subjected to linear regression, and the values of $r^{2}$ and the slopes of the regression lines were calculated. Pearson correlation coefficients (r) were analyzed for point-estimates and 95\% confidence intervals (CIs) using 2-tailed tests.

\section{Change of amplitude after administration of the $\alpha 1$-adrenoceptor antagonist}

For global analysis, amplitudes after administration of all a1adrenoceptor antagonists were compared to those before administration of the blockers by using a 2-tailed paired t-test. If the statistical significance was confirmed in the global analysis, the differences in changes of amplitude were compared between each $\alpha 1$-adrenoceptor antagonist in the same SG neuron, again using a 2-tailed paired t-test. Additionally, differences in the amplitudes before and after administration were compared for naftopidil, tamsulosin, and silodosin using the 2-tailed Tukey-Kramer test.

\section{Waterfall plot analysis for each $\alpha 1$-adrenoceptor blocker}

To compare the extent of changes in amplitudes by naftopidil, tamsulosin, and silodosin in individual SG neurons, waterfall plots were generated for the differences in amplitudes between the before-and after-administration values for each $\alpha 1$-adrenoceptor antagonist. The effects of each antagonist were plotted as histograms of values in descending order of difference.

\section{Inward and outward current of EPSC}

SG neurons that showed inward and/or outward currents in response to $\alpha 1$-adrenoceptor antagonist exposure were selected, and the corresponding data were used to calculate the rates of response.

\section{RESULTS}

\section{Whole-Cell Patch-Clamp Recordings From SG neurons}

Typical mEPSC recordings of changes of amplitude in response to $\alpha 1$-adrenoceptor antagonist exposure are provided in Fig. 2, including examples that exhibited changes in frequency and amplitude either without inducing outward and/or inward current (A), with inward current induced (B), or with outward current induced $(\mathrm{C})$.
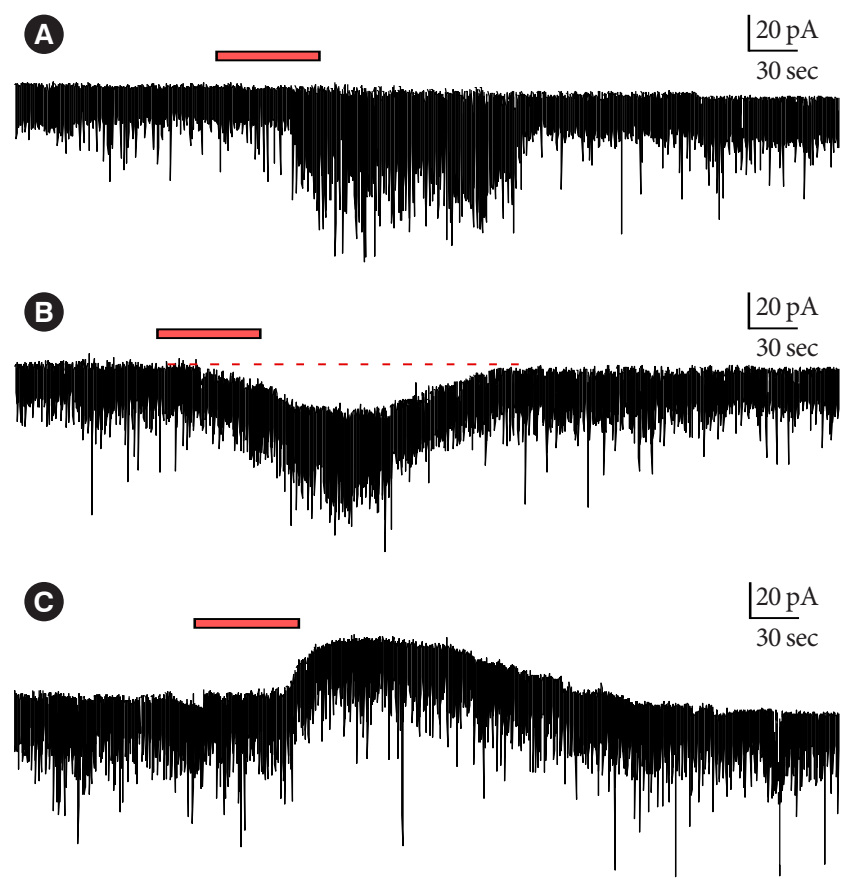

Fig. 2. Charts of typical mEPSCs are presented. (A) Frequency and amplitude were changed but outward/inward currents were not altered. (B, C) Inward current and outward currents (respectively) were observed. The bar indicates the time of application of the al-adrenoceptor blocker.

\section{Correlation of the Amplitudes Before and After Bath Application of Each $\alpha 1$-Adrenoceptor Antagonist in SG Neurons}

Pearson correlation coefficients for the amplitudes between the baseline and postadministration of $\alpha 1$-adrenoceptor antagonists ranked (in decreasing order) as naftopidil ( $r=0.393$; 95\% CI, 0.074-0.639), tamsulosin ( $\mathrm{r}=0.738$; 95\% CI, 0.541-0.859), and silodosin ( $r=0.944 ; 95 \% \mathrm{CI}, 0.891-0.971)$. For the correlation coefficient, the extent of lowering from 1.000 corresponds to potency of the drug. For naftopidil, tamsulosin, and silodo$\sin$, the regression lines yielded values for slopes of $0.573,1.046$, and 1.143, respectively (Fig. 3).

\section{Change of Amplitude After Administration of $\alpha 1$ - Adrenoceptor Antagonists}

Global analysis showed that the $\alpha 1$-adrenoceptor antagonists yielded significant increases in the amplitude of mEPSCs of SG neurons, which rose from $11.8 \mathrm{pA}$ at baseline to $13.0 \mathrm{pA}$ following exposure $(\mathrm{n}=108, \mathrm{P}=0.012)$ (Table 1$)$. However, only silodosin demonstrated a significant elevation (relative to the baseline) when the data were broken down by antagonist $(\mathrm{n}=$ 

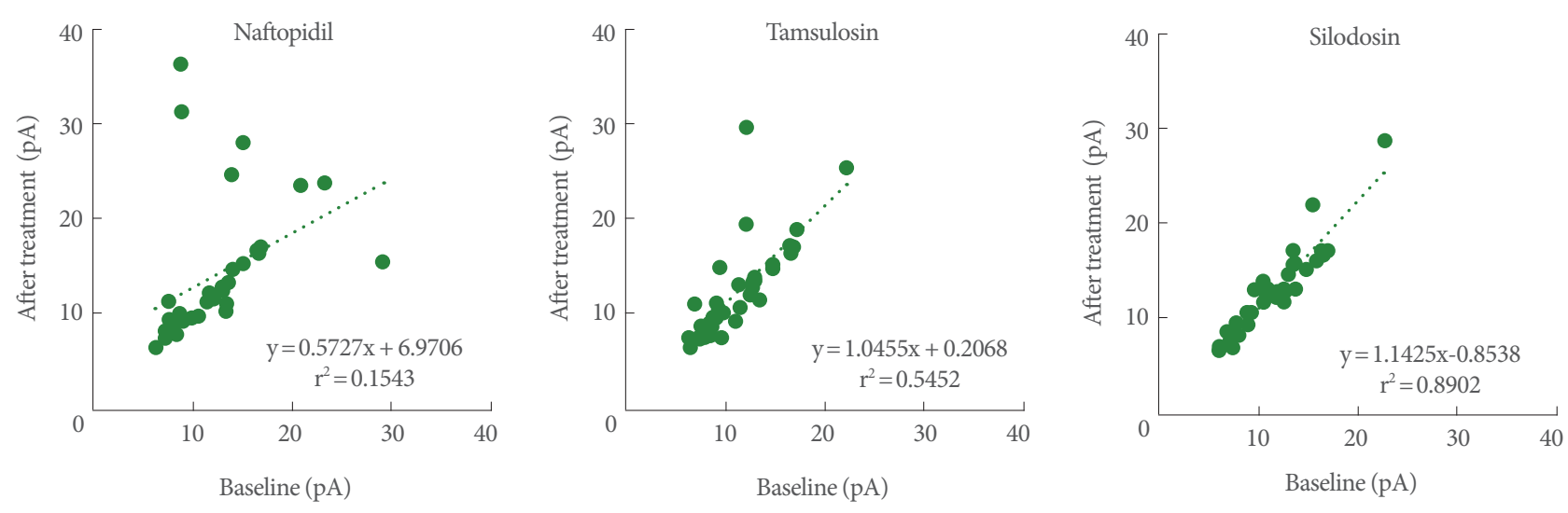

Fig. 3. Correlations of the amplitudes of mEPSCs between baseline and postadministration of $\alpha 1$-adrenoceptor antagonists. $\mathrm{n}=36$. mEPSC, miniature excitatory postsynaptic current.

Table 1. The effects of a1-adrenoceptor antagonists on the amplitude of EPSC in SG neurons of slices prepared from the lumbosacral level in rats

\begin{tabular}{|c|c|c|c|c|c|}
\hline \multirow{2}{*}{ a1-adrenoceptors } & \multicolumn{3}{|c|}{ Amplitude of EPSC, pA } & \multicolumn{2}{|r|}{ P-values } \\
\hline & Baseline & After treatment & $\Delta$ & vs. baseline & vs. drug \\
\hline Global (n=108) & $11.8 \pm 4.1(11.1-12.6)$ & $13.0 \pm 5.7(11.9-14.0)$ & $1.1 \pm 4.5(0.3-2.0)$ & 0.012 & - \\
\hline Naftopidil $(\mathrm{n}=36)$ & $12.1 \pm 4.9(10.5-13.8)$ & $13.9 \pm 7.1(11.5-16.3)$ & $1.8 \pm 6.9(-0.5$ to 4.1$)$ & 0.129 & $\begin{array}{l}0.226 \text {, vs. tamsulosin } \\
0.377 \text {, vs. silodosin }\end{array}$ \\
\hline Tamsulosin $(\mathrm{n}=36)$ & $11.7 \pm 3.6(10.5-12.9)$ & $12.5 \pm 5.0(10.8-14.2)$ & $0.7 \pm 3.4(-0.4$ to 1.9$)$ & 0.201 & 0.914 , vs. silodosin \\
\hline Silodosin $(\mathrm{n}=36)$ & $11.7 \pm 3.8(10.4-12.9)$ & $12.5 \pm 4.6(10.9-14.0)$ & $0.8 \pm 1.6(0.3-1.4)$ & 0.005 & - \\
\hline
\end{tabular}

Values are presented as means \pm standard error of the mean with $95 \%$ confidence interval in parentheses.

Statistical analyses were performed by 2-tailed paired Student $t$-tests to compare the values between baseline and after treatment in a given neuron, and by 2 -tailed Turkey-Kramer test to compare the values among the groups treated with distinct al-adrenoceptor antagonists.

EPSC, excitatory post synaptic current; SG, substantia gelatinosa.

36 each) from $11.7 \mathrm{pA}$ to $12.5 \mathrm{pA}(\mathrm{P}=0.005)$. Naftopidil and tamsulosin tended to increase the amplitudes but without statistical significance with individual effects as follows: naftopidil, from $12.1 \mathrm{pA}$ to $13.9 \mathrm{pA}(\mathrm{P}=0.129)$; tamsulosin, from $11.7 \mathrm{pA}$ to $12.5 \mathrm{pA}(\mathrm{P}=0.201)$ (Table 1). Additionally, statistical significances were not found among $\alpha 1$-adrenoceptor antagonists for the changes of amplitude before and after application of the antagonists to a given SG neuron (Table 1).

\section{Waterfall Plot Analysis for Each $\alpha$ 1-Adrenoceptor Antagonist}

As shown in Fig. 4, the waterfalls plots of naftopidil, tamsulosin, and silodosin permitted apparent ranking by the strength of the effects. The changes of amplitudes of mEPSCs for naftopidil ranged from positive to negative, while those for silodosin exhibited a narrower range.

\section{Inward and Outward Current of EPSCs}

Observability of inward or outward current following $\alpha 1$ adrenoceptor antagonist exposure differed among naftopidil (total: 9 out of 36 cells, 25.0\%; inward: 3 cells, 8.3\%; outward: 6 cells, 16.7\%), tamsulosin (total: 5 out of 36 cells, $13.9 \%$; outward only), and silodosin (total: 2 out of 36 cells, 5.6\%; outward only). The values of the currents are summarized in Table 2.

\section{DISCUSSION}

In individual SG neurons, each of $3 \alpha 1$-adrenoceptor antagonists was evaluated sequentially with $\mathrm{mEPSC}$ recording by the patch-clamp technique. The amplitudes of the mEPSCs were analyzed and the descriptive statistics values were noted. Various types of responsiveness to each $\alpha 1$-adrenoceptor antagonists were observed (Fig. 4). The responsiveness to naftopidil 


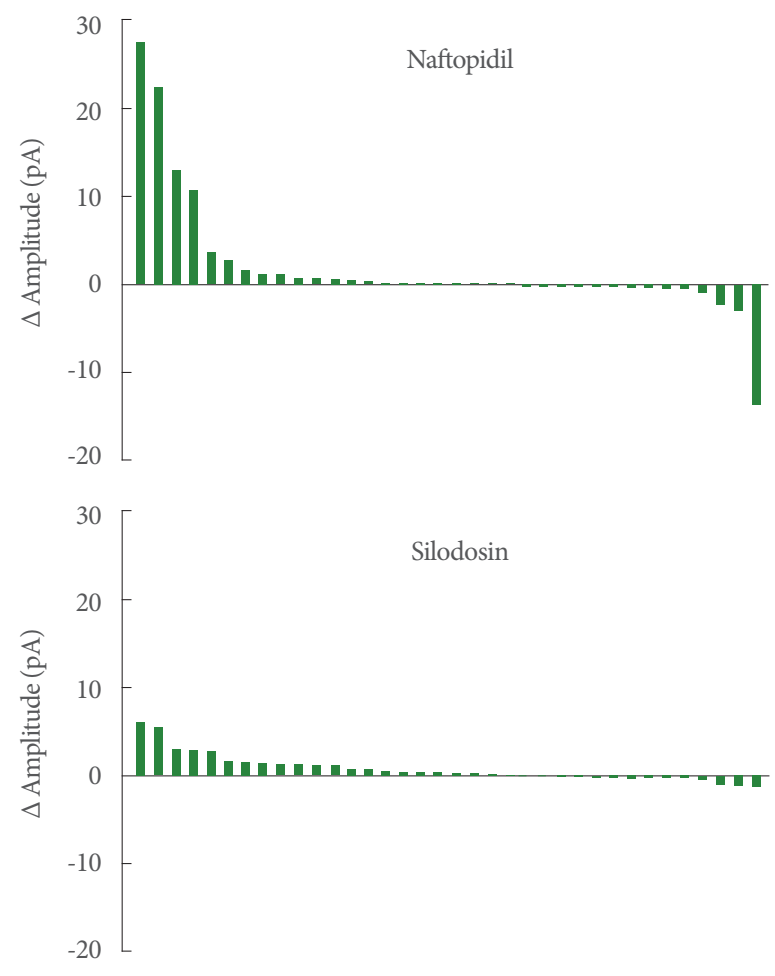

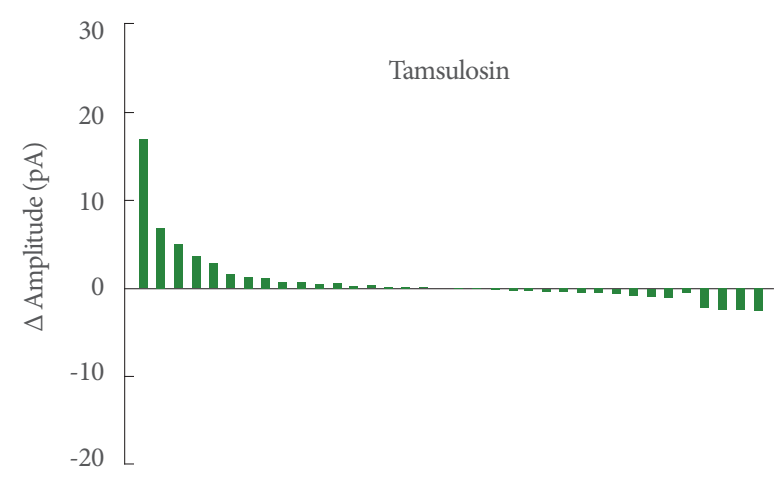

Fig. 4. Waterfall plots for the difference of amplitudes of mEPSCs between baseline and postadministration of al-adrenoceptor antagonists. The values of the differences were aligned in descending order. $\mathrm{n}=36$. $\mathrm{mEPSC}$, miniature excitatory postsynaptic current.

Table 2. The values of inward/outward currents generated by each a1-adrenoceptor antagonist

\begin{tabular}{|c|c|c|c|c|c|c|}
\hline \multirow{2}{*}{ ID } & \multicolumn{2}{|c|}{ Naftopidil (pA) } & \multicolumn{2}{|c|}{ Tamsulosin (pA) } & \multicolumn{2}{|c|}{ Silodosin (pA) } \\
\hline & Inward & Outward & Inward & Outward & Inward & Outward \\
\hline 1 & 0 & 29.42 & 0 & 7.99 & 0 & 10.44 \\
\hline 7 & 5.63 & 0 & 0 & 0 & 0 & 0 \\
\hline 8 & 0 & 15.94 & 0 & 6.66 & 0 & 0 \\
\hline 9 & 0 & 9.61 & 0 & 6.18 & 0 & 0 \\
\hline 10 & 0 & 10.24 & 0 & 8.12 & 0 & 7.34 \\
\hline 12 & 0 & 10.74 & 0 & 2.22 & 0 & 0 \\
\hline 13 & 12.65 & 0 & 0 & 0 & 0 & 0 \\
\hline 19 & 0 & 16.46 & 0 & 0 & 0 & 0 \\
\hline 21 & 10.20 & 0 & 0 & 0 & 0 & 0 \\
\hline No. (\%) & $3(8.3)$ & $6(16.7)$ & $0(0)$ & $5(13.9)$ & $0(0)$ & $2(5.6)$ \\
\hline
\end{tabular}

was twice that to tamsulosin (25\% vs. $13.9 \%$ ), and 5 times that to silodosin ( $25 \%$ vs. $5.6 \%$ ), as shown in Table 2.

\section{Correlation of the Amplitude of mEPSCs Between Baseline and After Application of Each a1-Adrenoceptor Antagonist}

For tamsulosin and silodosin, Pearson correlation coefficients for the amplitudes between baseline and after the application were 0.738 and 0.944 (respectively), implying strong correlation. In contrast, the value for naftopidil was 0.393 , suggesting weak correlation. These results indicated that the changes of amplitude of mEPSCs between before and after the application of $\alpha 1$-adrenoceptor antagonists are small for tamsulosin or silodosin and large for naftopidil. Furthermore, since a change in the amplitude of mEPSCs reveals a change in sensitivity for postsynaptic excitation (e.g., an upregulation of receptors for glutamate), naftopidil may promote changes in sensitivity, an effect not seen with tamsulosin and silodosin. 


\section{Responsiveness of the Amplitude of mEPSCs to 1 1- Adrenoceptor Antagonists}

Our global analysis indicated that $\alpha 1$-adrenoceptor antagonists increase the amplitude of the mEPSCs of SG neurons. We hypothesize that the effects of naftopidil and tamsulosin were not statistically significant because the numbers of SG neurons that responded to naftopidil or tamsulosin were low. For instance, if the responsive SG neurons are defined as those in which the amplitude (pA) of mEPSCs change by $>20 \%$ or $<-20 \%$ (relative to the baseline), then 8 would be considered responsive to naftopidil and 5 each would be considered responsive to tamsulosin or silodosin (out of 36 SG neurons each; data not shown). In terms of the differences $(\Delta)$ in amplitude, the effect size was smallest in the silodosin group (Table 1). Therefore, we infer that the smaller variance in the amplitude of mEPSCs was the reason that statistical significance was observed after application of silodosin. The ranges of responses observed in the amplitudes differed among the $\alpha 1$-adrenoceptor antagonists, as shown in the waterfall plots (Fig. 3). The waterfall plots for tamsulosin and silodosin resembled each other, which may reflect the small difference in the Pearson correlation coefficients for these 2 antagonists ( 0.738 and 0.944, respectively). Although the increases in the amplitude of mEPSCs with naftopidil and tamsulosin fell short of statistical significance, the responsive SG neurons (i.e., those whose amplitude (in pA) increased or decreased by more than $20 \%$ relative to the baseline) represented a subpopulation (among 36 SG neurons each) of 8 for naftopidil and 5 for tamsulosin neurons (data not shown). These results suggested that the neurons responsive to specific $\alpha 1$ adrenoreceptor blockers are present in defined proportions. Given that mEPSCs represent glutamatergic transmission in afferent fibers [20], increases in the amplitude of mEPSCs suggest upregulation of the sensitivity of receptors that mediate glutamatergic transmission, which in turn would be expected to facilitate inhibitory GABAergic and/or glycinergic interneurons. Although silodosin exposure yielded a significant increase in the amplitude of mEPSCs (Table 1), the magnitude of the effect was small. Therefore, silodosin may have a limited effect on SG neurons.

Previous work showed that naftopidil suppresses the amplitude of mEPSCs evoked by dorsal root stimulation in the SG neurons of slices isolated from the lumbosacral spine, while prazosin does not [21]. Those results indicated that naftopidil may attenuate the excitation caused by primary afferent fibers at the postsynaptic site without blocking a1-adrenoceptors.
Furthermore, naftopidil facilitated larger changes in the frequencies of mEPSCs in SG neurons of slices isolated from the same level of spine than did tamsulosin or silodosin [17]. This distinction suggests that naftopidil's ability to suppress the micturition reflex may result from excitation of postsynaptic cells via an elevated frequency of neurotransmitter release from the presynaptic sites, facilitating inhibition in subsequent inhibitory interneurons. The present study indicated that naftopidil increased and decreased the amplitude of the mEPSCs in SG neurons (Fig. 4). The increases suggest upregulation of the sensitivity at the postsynaptic site for the neurotransmitter (e.g., GABA, glycine) released from the presynaptic terminal. The decreases suggest downregulation of the sensitivity at the postsynaptic site for the neurotransmitter (e.g., glutamate) released from the presynaptic terminal. These bidirectional effects suggest that increases and decreases in amplitude may reflect the excitation and sedation (respectively) of postsynaptic cells.

In the present study, the a1-adrenoceptor antagonists generated inward or outward currents in a subset of SG neurons; notably, the outward current was observed more readily than was the inward current (Table 2). We speculate that the 3 antagonists directly facilitate hyperpolarization of SG neurons by generating outward currents. In view of the inward current seen for mEPSCs, it is possible that naftopidil encourages depolarization in a small subpopulation of the SG neurons. These results suggest that naftopidil modulates neurotransmission in the SG neurons multimodally.

\section{Extrapolation of Changes in mEPSCs to Effects on Micturition}

It remains difficult, using the current technique, to determine an actual relationship between the amplitude of EPSC and the storage symptoms. At minimum, in vivo electrophysiological recording would need to be combined with cystometry, which would have to be performed under anesthesia. As far as we know, correlations between urinary bladder activity and afferent nerve activity, but not between bladder activity and EPSC, have been reported, in which action potentials of afferent fibers isolated from the left L6 dorsal root were recorded [22]. In the previous study on EPSC, a correlation between the amplitude and the afferent nociceptive input was reported [19]. There is a substantial difference between painful stimuli and storage symptoms, but both are aspects of the afferent sensory nerve. Therefore, we speculate that the amplitude of EPSC may modulate the storage symptoms. 
During the storage of urine, the storage reflex generated by primary afferent firing is coordinated by the spinal reflex pathway [23]. In human functional analysis, bladder activity is mediated by stimulation of brain subregions (for example, the periaqueductal gray matter [24]). In the lumbosacral region, the SG (lamina II) of the spinal dorsal horn contains a high density of excitatory and inhibitory interneurons that are thought to be critically involved in the modulation of nociception [25] and (presumably) the micturition reflex. Sensory information is carried from the pelvic organs to the dorsal horn of the lumbosacral spinal cord [26]. In the rat spinal cord, glutamatergic mechanisms play an essential role in micturition control [27]. Intrathecal injection of a1-adrenoceptor antagonists has been shown to inhibit the micturition reflex in animal models $[7,28]$. These results suggest that the al-adrenoceptor antagonists exert their activity at the spinal level in the neural circuitry. In the present study, several neurons exhibited increased or decreased amplitudes of mEPSCs in response to a1adrenoceptor antagonists, particularly naftopidil (Fig. 4). These results indicated that the postsynaptic sites of various SG neurons have differing sensitivities for excitatory neurotransmitters (e.g., glutamate). Although the physiological significance of the amplitude of the EPSC has not been determined to date, the 3 a1-adrenoceptor antagonists tested in the present study are presumed to exert their activities in the postsynaptic site, while prazosin apparently does not [21]. As shown in the waterfall plots of the present study, neurons that responded to the a1adrenoceptor antagonists predominantly exhibited increased (and not decreased) amplitudes of mEPSCs. Therefore, we speculate that naftopidil and tamsulosin suppress micturition by upregulating sensitivity at the postsynaptic site of inhibitory interneurons, thereby activating inhibitory neurotransmission. Additionally, silodosin may contribute in part by activating the inhibitory pathway in the spinal cord.

In conclusion, although exposure to a1-adrenoceptor antagonists increased the amplitudes of mEPSCs in rat SG neurons in slices prepared from the L6-S1 spine level, these effects reflected responses in a subset of the SG neurons. Additionally, the a1-adrenoceptor antagonists generated inward/outward currents in the SG neurons. The magnitude of the changes induced by the al-adrenoceptor antagonists ranged (in descending order) from naftopidil to tamsulosin to silodosin. These results are expected to increase our understanding of the mechanisms of action (at the spine level) of a1-adrenoceptor antagonists when used clinically for the treatment of storage symp- toms associated with LUTS.

This study is phenomenologically based; therefore, further electrophysiological and molecular biological investigations of the proposed mechanisms will be needed. Although afferent nerves from the urothelium largely project to lamina $\mathrm{X}$ in the dorsal horn, SG neurons in lamina II were used. As described previously, a part of the superficial neurons receives nociceptive and non-nociceptive inputs from the lower urinary tract, as shown by upregulation of cFos expression [29]. Although the use of lamina $\mathrm{X}$ would be ideal, that structure possesses a low density of neurons, making the blind attachment of patchclamp electrodes less efficient. Therefore, lamina II was examined. Furthermore, our results indicated that a1-adrenoceptor antagonists alter the amplitudes of mEPSCs in only a subset of SG neurons. This observation is consistent with the existence of multiple types of SG neurons within this lumbosacral level [30].

\section{AUTHOR CONTRIBUTION STATEMENT}

- Conceptualization: $D U, M Y$

- Formal Analysis: DU, TH

- Investigation: $D U, T H$

- Methodology: DU, TH

- Project Administration: $T H$

- Writing - Original Draft: $D U, T H$

- Writing - Review \& Editing: $D U, T H$

\section{REFERENCES}

1. Abrams P, Cardozo L, Fall M, Griffiths D, Rosier P, Ulmsten U, et al. The standardisation of terminology in lower urinary tract function: report from the standardisation sub-committee of the International Continence Society. Urology 2003;61:37-49.

2. Management of non-neurogenic male LUTS [Internet]. Arnhem (The Netherlands): European Association of Urology; 2018 [cited 2018 Dec 8]. Available from: https://uroweb.org/guideline/treatment-ofnon-neurogenic-male-luts/.

3. Rohrmann S, Katzke V, Kaaks R. Prevalence and progression of lower urinary tract symptoms in an aging population. Urology 2016;95:158-63.

4. Takei R, Ikegaki I, Shibata K, Tsujimoto G, Asano T. Naftopidil, a novel alpha1-adrenoceptor antagonist, displays selective inhibition of canine prostatic pressure and high affinity binding to cloned human alpha1-adrenoceptors. Jpn J Pharmacol 1999;79:447-54.

5. Kwon SY, Lee KS, Yoo TK, Chung JI, Lee JY, Hong JH, et al. Com- 
parison of the effect of naftopidil $75 \mathrm{mg}$ and tamsulosin $0.2 \mathrm{mg}$ on the bladder storage symptom with benign prostatic hyperplasia: prospective, multi-institutional study. Urology 2018;111:145-50.

6. Chen Z, Ishizuka O, Imamura T, Aizawa N, Igawa Y, Nishizawa O, et al. Role of alphal-adrenergic receptors in detrusor overactivity induced by cold stress in conscious rats. Neurourol Urodyn 2009;28:251-6.

7. Sugaya K, Nishijima S, Miyazato M, Ashitomi K, Hatano T, Ogawa Y. Effects of intrathecal injection of tamsulosin and naftopidil, alpha-1A and -1D adrenergic receptor antagonists, on bladder activity in rats. Neurosci Lett 2002;328:74-6.

8. Sugaya K, Nishijima S, Kadekawa K, Ashitomi K, Ueda T, Yamamoto H. Spinal mechanism of micturition reflex inhibition by naftopidil in rats. Life Sci 2014;116:106-11.

9. Gotoh M, Kamihira O, Kinukawa T, Ono Y, Ohshima S, Origasa H, et al. Comparison of tamsulosin and naftopidil for efficacy and safety in the treatment of benign prostatic hyperplasia: a randomized controlled trial. BJU Int 2005;96:581-6.

10. Nishino Y, Masue T, Miwa K, Takahashi Y, Ishihara S, Deguchi T. Comparison of two alpha1-adrenoceptor antagonists, naftopidil and tamsulosin hydrochloride, in the treatment of lower urinary tract symptoms with benign prostatic hyperplasia: a randomized crossover study. BJU Int 2006;97:747-51, discussion 751.

11. Rexed B. The cytoarchitectonic organization of the spinal cord in the cat. J Comp Neurol 1952;96:414-95.

12. Yang K, Feng Y, Li Y. Baclofen inhibition of dorsal root-evoked inhibitory postsynaptic currents in substantia gelatinosa neurons of rat spinal cord slice. Brain Res 2001;900:320-3.

13. Yang K, Kumamoto E, Furue H, Li YQ, Yoshimura M. Action of capsaicin on dorsal root-evoked synaptic transmission to substantia gelatinosa neurons in adult rat spinal cord slices. Brain Res 1999;830:26873.

14. Ikeda K, Satake S, Onaka T, Sugimoto H, Takeda N, Imoto K, et al. Enhanced inhibitory neurotransmission in the cerebellar cortex of Atpla3-deficient heterozygous mice. J Physiol 2013; 591:3433-49.

15. Xu ZH, Wang C, Fujita T, Jiang CY, Kumamoto E. Action of thymol on spontaneous excitatory transmission in adult rat spinal substantia gelatinosa neurons. Neurosci Lett 2015; 606:94-9.

16. Yang K, Li YQ. Origins of spontaneous and noxious stimuli-evoked miniature EPSCs in substantia gelatinosa. Neuroreport 2001; 12:39-42.

17. Uta D, Hattori T, Yoshimura M. Characterization on responsiveness of excitatory synaptic transmissions to al-adrenoceptor blockers in substantia gelatinosa neurons isolated from lumbo- sacral level in rat spinal cords. Int Neurourol J 2019; 23:13-21.

18. Uta D, Xie DJ, Hattori T, Kasahara KI, Yoshimura M. Effects of naftopidil on inhibitory transmission in substantia gelatinosa neurons of the rat spinal dorsal horn in vitro. J Neurol Sci 2017;380:205-11.

19. Uta D, Kato G, Doi A, Andoh T, Kume T, Yoshimura M, et al. Animal models of chronic pain increase spontaneous glutamatergic transmission in adult rat spinal dorsal horn in vitro and in vivo. Biochem Biophys Res Commun 2019;512:352-9.

20. Guo YX, Li DP, Chen SR, Pan HL. Distinct intrinsic and synaptic properties of pre-sympathetic and pre-parasympathetic output neurons in Barrington's nucleus. J Neurochem 2013;126:338-48.

21. Uta D, Hattori T, Yoshimura M. Effects of high concentrations of naftopidil on dorsal root-evoked excitatory synaptic transmissions in substantia gelatinosa neurons in vitro. Int Neurourol J 2018;22:252-9.

22. Aizawa N, Sugiyama R, Ichihara K, Fujimura T, Fukuhara H, Homma Y, et al. Functional roles of bladder al-adrenoceptors in the activation of single-unit primary bladder afferent activity in rats. BJU Int 2016;117:993-1001.

23. de Groat WC. Integrative control of the lower urinary tract: preclinical perspective. Br J Pharmacol 2006;147 Suppl 2(Suppl 2):S25-40.

24. Griffiths D, Derbyshire S, Stenger A, Resnick N. Brain control of normal and overactive bladder. J Urol 2005;174:1862-7.

25. Todd AJ. Neuronal circuitry for pain processing in the dorsal horn. Nat Rev Neurosci 2010;11:823-36.

26. Brown AG. The dorsal horn of the spinal cord. Q J Exp Physiol 1982;67:193-212.

27. Yoshiyama M, de Groat WC. Supraspinal and spinal alpha-amino3-hydroxy-5-methylisoxazole-4-propionic acid and N-methyl-Daspartate glutamatergic control of the micturition reflex in the urethane-anesthetized rat. Neuroscience 2005;132:1017-26.

28. Yoshizumi M, Matsumoto-Miyai K, Yonezawa A, Kawatani M. Role of supraspinal and spinal alphal-adrenergic receptor subtypes in micturition reflex in conscious rats. Am J Physiol Renal Physiol 2010;299:F785-91.

29. Birder LA, de Groat WC. Increased c-fos expression in spinal neurons after irritation of the lower urinary tract in the rat. J Neurosci 1992;12:4878-89.

30. Uta D, Furue H, Pickering AE, Rashid MH, Mizuguchi-Takase H, Katafuchi T, et al. TRPA1-expressing primary afferents synapse with a morphologically identified subclass of substantia gelatinosa neurons in the adult rat spinal cord. Eur J Neurosci 2010;31:1960-73. 